\title{
Reduction of multi-stage disk models: Application to an industrial rotor
}

\author{
Arnaud Sternchüss* and Etienne Balmès \\ Laboratoire MSSMAT, École Centrale Paris, \\ Grande Voie des Vignes, 92295 Châtenay-Malabry Cedex, France \\ Pierrick Jean and Jean-Pierre Lombard \\ Snecma (Safran Group), Site de Villaroche, \\ Rond-point René Ravaud, 77550 Moissy-Cramayel Cedex, France
}

\begin{abstract}
The present study deals with the reduction of models of multi-stage bladed disk assemblies. The proposed method relies on the substructuring of the rotor into sectors. The bladed disks are coupled by intermediate rings which remove the problem of incompatible meshes. The sectors are represented by super-elements whose kinematic subspaces are spanned by a set of cyclic modeshapes and a set of normal modes when their left and right interfaces are fixed. The first step is to compute the cyclic modeshapes that are defined on the full rotor by enforcing the uncoupling of the spatial Fourier harmonics. This leads to a family of subproblems parameterized by the harmonic coefficient, similarly to the classical approach used to deal with tuned bladed disks. The subsequent reduction process leads to compact reduced models whose accuracy has been extensively tested on simple but realistic academic models. The proposed methodology was then applied to an industrial rotor to conduct an analysis at a wider scale. This case was also the occasion to point out the fact that the assembly of individual disk models into a rotor model is really straightforward and provides an efficient tool to observe and predict coupled phenomena.
\end{abstract}

\footnotetext{
${ }^{*}$ Corresponding author: arnaud.sternchuss@ecp.fr 


\section{INTRODUCTION}

This study presents an industrial application of the authors' reduction technique to determine the modal characteristics of assemblies of bladed disks.

The current industrial practice is to model separately each bladed disk of the rotor. When such disks are cyclically symmetric, their dynamics are fully determined from that of a small portion, typically a bladed sector. This configuration no longer holds in real rotors due to mistuning which significantly alters this behaviour since it can strongly localize the strain energy $^{1}$. Therefore, full-scale analyses require huge models, all the more since many nearly identical computations are needed to cover the entire space of parameters (mistuning coefficients, rotation speed, temperature,...). To address this issue, many reduction techniques have been developed such as those of Lim et al. ${ }^{2}$, Feiner and Griffin ${ }^{3,4}$ and Sternchüss et al. ${ }^{5}$, among others, that rely on synthesis techniques using either component or system modes.

Such reduced representations would be a good starting point for dealing with multi-stage rotors. However, as underlined by Bladh et $a l^{6}{ }^{6}$, the critical point is the choice of the boundary conditions that would properly represent inter-stage coupling, which is the main cause of discrepancies between single stage and multi-stage dynamics, since this coupling ratio controls the spread of the strain energy over many disks. Song et al. ${ }^{7}$, Laxalde et al. ${ }^{8}$, and Sternchüss and Balmès ${ }^{9}$ proposed recently efficient CMS techniques that include such coupling directly into the formulation of the reduced problem.

The first part of this paper updates the methodology developed in ${ }^{9}$. This method consists in a decomposition of the disks into sector super-elements connected with each other through inter-sector elements and inter-disk ring elements. The updates mostly concern the formulation of the problems that lead to the cyclic modeshapes as defined by Laxalde et $a l .{ }^{8}$, and the process used to generate the subspaces in which the kinematics of the sector super-elements are sought. The assembly of the problem remains really straightforward, leading to a very compact model whose accuracy is driven by the choice of the reduction bases. The main results obtained for a simple academic model that evaluates the accuracy of the proposed technique are described throughout this section.

The second part of this paper then presents the application of the methodology to a subset of a realistic industrial rotor provided by Snecma. From these results, the concluding section provides some guidelines for the practical use of such a reduction method in a global 
design process.

\section{Nomenclature}

$\mathcal{I}$ Interface

$\mathcal{D} \quad$ Disk

$\mathcal{R} \quad$ Inter-disk ring

$\mathcal{S}$ Sector

[c] Observation matrix

$[E]$ Harmonic recovery matrix

$[I] \quad$ Identity matrix

$[K]$ Stiffness matrix

$[M]$ Mass matrix

[Z] Dynamic stiffness matrix

$[\Phi]$ Matrix of eigenvectors

$[T]$ Basis of a subspace

$\left\{f_{e}\right\}$ External load vector

$\{q\}$ DOF vector

$\{\widehat{q}\}$ Harmonic DOF vector

$\alpha \quad$ Sector angle

$\delta \quad$ Fourier harmonic

i $\sqrt{-1}$

$\omega \quad$ Natural frequency

$f \quad$ Normalized frequency

$N \quad$ Number of degrees of freedom

$N_{s} \quad$ Number of sectors

$R$ Participation factor in strain energy

* Complex conjugate

$*_{a} \quad$ Quantity relative to aft interface

$*_{f} \quad$ Quantity relative to front interface

$*_{c} \quad$ Quantity relative to complementary domain 
$*_{l} \quad$ Quantity relative to left interface

$*_{r} \quad$ Quantity relative to right interface

$*_{M} \quad$ Quantity relative to multi-stage reduction

* $\quad$ Quantity relative to harmonic $\delta$

$*^{d} \quad$ Quantity relative to disk $d$

$*^{d, s} \quad$ Quantity relative to sector $s$ of disk $d$

$*^{\mathcal{R}} \quad$ Quantity relative to inter-disk ring $\mathcal{R}$

CMS Component Mode Synthesis

DOF Degree of Freedom

MAC Modal Assurance Criterion

\section{MULTI-STAGE REDUCTION AT FIXED SPEED}

The considered structure is presented in Fig. 1. It is composed of two bladed disks nominally tuned $\mathcal{D}^{1}$ and $\mathcal{D}^{2}$ with 12 and 15 blades respectively connected with a volumic interface $\mathcal{R}$. The disks are meshed with brick elements and the ring is meshed with tetrahedrons obtained by a Delaunay tessellation (MATLAB function delaunay3) between the nodes of the aft rim of disk $\mathcal{D}^{1}$, denoted $\mathcal{I}_{a}^{1}$, and the front rim of disk $\mathcal{D}^{2}$, denoted $\mathcal{I}_{f}^{2}$. The material is titanium whose properties are $E=120 \mathrm{GPa}, \nu=0.33$ and $\rho=4700 \mathrm{~kg} \cdot \mathrm{m}^{-3}$. The radii at the roots of the blades are $200 \mathrm{~mm}$ and $205 \mathrm{~mm}$ respectively, the radii at their tips are $280 \mathrm{~mm}$ and $285 \mathrm{~mm}$ respectively. Their thickness varies from $6 \mathrm{~mm}$ at the root to $2 \mathrm{~mm}$ at the top. Their widths are $43 \mathrm{~mm}$ and $46 \mathrm{~mm}$ at the top. The frequency bandwidth of interest for this study is $[0,14]$, if all frequencies are normalized with respect to the lower flexible natural frequency of the rotor.

\section{Coupled mechanical problem}

The meshes of the aft rim $\mathcal{I}_{a}^{1}$ of $\mathcal{D}^{1}$ and the front rim $\mathcal{I}_{f}^{\mathcal{R}}$ of $\mathcal{R}$ are identical to avoid problems of incompatibility. For the same reasons, the meshes of the aft $\operatorname{rim} \mathcal{I}_{a}^{\mathcal{R}}$ of $\mathcal{R}$ and the front $\operatorname{rim} \mathcal{I}_{f}^{2}$ of $\mathcal{D}^{2}$ are identical. Due to the way it is meshed, the inter-disk ring $\mathcal{R}$ has a single element along the $\mathbf{z}$ direction, thus it has no interior DOF when it is made of first order elements. $\mathcal{D}^{1}$ and $\mathcal{D}^{2}$ have $N^{1}$ and $N^{2}$ DOF respectively. These two DOF sets 


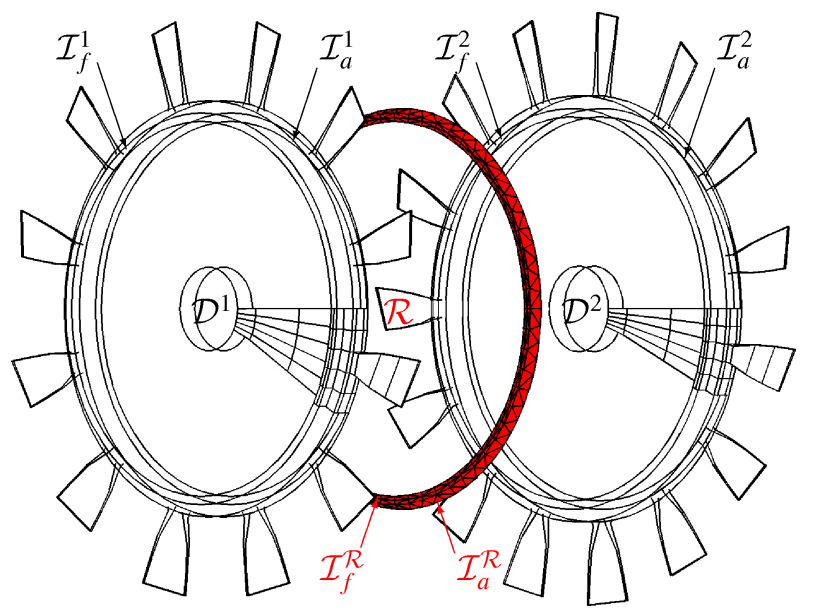

FIG. 1: Sample assembly

are disjoint. The total number of DOF is $N=N^{1}+N^{2}$. The discrete displacement $\{q\}$ is continuous at the interface between two adjacent substructures:

$$
\left\{q_{a}^{1}\right\}=\left\{q_{f}^{\mathcal{R}}\right\} \text { and }\left\{q_{f}^{2}\right\}=\left\{q_{a}^{\mathcal{R}}\right\}
$$

The particular geometry of $\mathcal{R}$ implies that its DOF are fully determined by $\left\{q^{\mathcal{R}}\right\}=$ $\left\{q_{f}^{\mathcal{R}} q_{a}^{\mathcal{R}}\right\}^{\top}$.

In the frequency domain, if an external load $\left\{f_{e}(\omega)\right\}$ is given, the discrete displacement $\{q(\omega)\}$ satisfies:

$$
[Z(\omega)]_{N \times N}\{q(\omega)\}_{N \times 1}=\left\{f_{e}(\omega)\right\}_{N \times 1}
$$

The dynamic stiffness matrix $[Z]$ of the rotor is

$$
[Z(\omega)]=\left[\begin{array}{cc}
Z^{1}(\omega) & 0 \\
0 & Z^{2}(\omega)
\end{array}\right]+\left[Z^{\mathcal{R}}(\omega)\right]
$$

where $\left[Z^{1}\right],\left[Z^{\mathcal{R}}\right]$ and $\left[Z^{2}\right]$ stand for the dynamic stiffness matrices of $\mathcal{D}^{1}, \mathcal{R}$ and $\mathcal{D}^{2}$ respectively.

\section{Spatial harmonic content}

Let us consider a single disk $\mathcal{D}^{d}$ in its nominal configuration. Under the assumption of cyclic symmetry, it is made of $N_{s}^{d}$ identical angular sectors, numbered from 0 to $N_{s}^{d}-1$, generated by successive rotations of a reference sector. Let $\theta^{d}$ be the rotation of angle 
$\alpha^{d}=2 \pi / N_{s}^{d}$ around the $\mathbf{z}$ axis. Since the discrete geometry is invariant whatever the rotation $\left(\theta^{d}\right)^{s}$, the discrete displacement $\left\{q^{d, s}\right\}$ is paired with its Fourier harmonic coefficients $\left\{\widehat{q}_{\delta}^{d}\right\}$ such that, in the local basis of sector $\mathcal{S}^{d, s}$, it is written ${ }^{5,10}$ :

$$
\forall s \in \mathbb{N}_{N_{s}^{d}-1},\left\{q^{d, s}\right\}=\Re\left(\sum_{\delta=0}^{N_{s}^{d} / 2}\left\{\widehat{q}_{\delta}^{d}\right\} e^{\mathbf{i} s \delta \alpha^{d}}\right)
$$

The spatial harmonic DOF vectors $\left\{\widehat{q}_{\delta}^{d}\right\}$ are defined on the reference sector $\mathcal{S}^{d, 0}$ and are complex quantities, except for $\delta=0$ and $\delta=N_{s}^{d} / 2$, if $N_{s}^{d}$ is even, multiplied by a dephasing factor that depends on the harmonic coefficient $\delta$ and the sector number $s$. Throughout the rest of the paper, the terms associated with harmonic coefficient $N_{s}^{d} / 2$ are systematically kept, even if $N_{s}^{d}$ is odd, in order to avoid dealing with different cases.

As stated by Eqn. (4), there exists a duality between the physical DOF vector $\left\{q^{d}\right\}$ and the harmonic DOF vector $\left\{\widehat{q}^{d}\right\}$ :

$$
\begin{aligned}
& \left\{q^{d}\right\}=\left\{\begin{array}{llll}
q^{d, 0} & q^{d, 1} q^{d, 2} \cdots q^{d, N_{s}-1}
\end{array}\right\}^{\top} \\
& \left\{\widehat{q}^{d}\right\}=\left\{\widehat{q}_{0}^{d} \Re\left(\widehat{q}_{1}^{d}\right) \Im\left(\widehat{q}_{1}^{d}\right) \cdots \Re\left(\widehat{q}_{N_{s}^{d} / 2-1}^{d}\right) \Im\left(\widehat{q}_{N_{s}^{d} / 2-1}^{d}\right) \widehat{q}_{N_{s}^{d} / 2}^{d}\right\}^{\top}
\end{aligned}
$$

$\Re$ and $\Im$ stand for the real and imaginary part of a complex quantity. The relation between these DOF vectors is

$$
\left\{q^{d}\right\}=\left[E^{d} \otimes I_{N^{d, 0}}\right]\left\{\widehat{q}^{d}\right\}
$$

where $\left[I_{N^{d, 0}}\right]$ is the identity matrix whose size is the number of physical DOF of sector $\mathcal{S}^{d, 0}$ and $\otimes$ is the Kronecker product.The block-column $\left[E_{\delta}^{d}\right]$ that corresponds to coefficient $\delta$ is

$$
\begin{aligned}
{\left[E_{0}^{d}\right] } & =\left[\begin{array}{lllll}
1 & \cdots & 1 & \cdots & 1
\end{array}\right]^{\top} \\
{\left[E_{\delta}^{d}\right] } & =\left[\begin{array}{cccccc}
2 & 2 \cos \left(\delta \alpha^{d}\right) & \cdots & 2 \cos \left(s \delta \alpha^{d}\right) & \cdots & 2 \cos \left(\left(N_{s}^{d}-1\right) \delta \alpha^{d}\right) \\
0 & -2 \sin \left(\delta \alpha^{d}\right) & \cdots & -2 \sin \left(s \delta \alpha^{d}\right) & \cdots & -2 \sin \left(\left(N_{s}^{d}-1\right) \delta \alpha^{d}\right)
\end{array}\right]^{\top} \\
{\left[E_{N_{s}^{d} / 2}^{d}\right] } & =\left[\begin{array}{lllll}
1 & \cdots & (-1)^{s} & \cdots & (-1)^{N_{s}^{d}-1}
\end{array}\right]^{\top}
\end{aligned}
$$

When dealing with an assembly of two disks, the assumption about the ring states that any physical DOF belongs either to disk $\mathcal{D}^{1}$ or to disk $\mathcal{D}^{2}$. Therefore, the global DOF vector $\{q\}$ is

$$
\{q\}=\left[\begin{array}{cc}
E^{1} \otimes I_{N^{1,0}} & 0 \\
0 & E^{2} \otimes I_{N^{2,0}}
\end{array}\right]\{\widehat{q}\}
$$


where $\{q\}=\left\{\begin{array}{ll}q^{1} & q^{2}\end{array}\right\}^{\top}$ and $\{\widehat{q}\}=\left\{\begin{array}{ll}\widehat{q}^{1} & \widehat{q}^{2}\end{array}\right\}^{\top}$.

Thanks to this, the spectral content of the modes of the whole rotor can be determined relatively to each disk. Figure 2 displays the result for the 74 first flexible modes of the sample rotor. For a given mode and a given disk $\mathcal{D}^{d}$, the amplitudes of the harmonic DOF vectors are normalized such that

$$
\max _{\delta \in\left\{0, N_{s}^{d} / 2\right\}}\left\|\widehat{q}_{\delta}^{d}\right\| \|_{1}=1
$$

which allows to isolate the dominating harmonic. This figure also clearly shows, on the one hand, that the modes come in families and suggests, on the other hand, that they come in pairs. A closer look at the frequencies associated with these near-pairs of modes indicates that they are very close but different. Moreover, this figure confirms the fact that some modes are multi-harmonic.

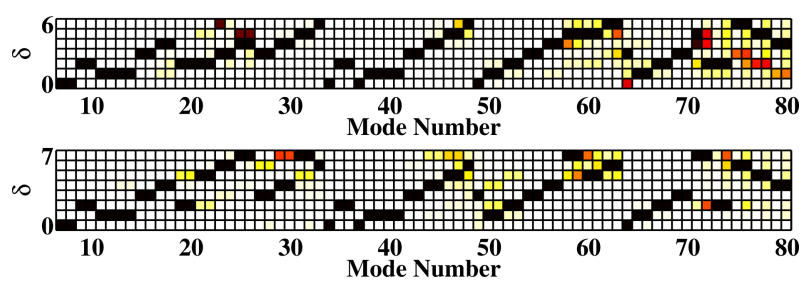

FIG. 2: Spatial spectrum of the 74 first flexible modes of the sample rotor

With the previous diagram, some particular modes can be identified, as described in Fig. 3. The harmonic DOF vectors are here normalized such that

$$
\max _{d \in\{1,2\}}\left(\max _{\delta \in\left\{0, N_{s}^{d} / 2\right\}}\left\|\widehat{q}_{\delta}^{d}\right\|_{1}\right)=1
$$

One then finds mono-harmonic coupled modes, like the near-pair of modes at $f=4.25$ and $f=4.26$ that seems to correspond to $\delta=2$. Thus, the ring participates in theses modes, but its lack of symmetry does not affect the motion very much. On the contrary, the mode at $f=2.99$ which is localized to disk $\mathcal{D}^{1}$ is multi-harmonic and the perturbation lies in the asymmetry of the ring, even though its participation is small.

\section{Mono-harmonic eigensolutions}

From the previous study of the complete rotor, the main conclusion to be drawn is that modes are not always mono-harmonic. According to this, mono-harmonic solutions such 


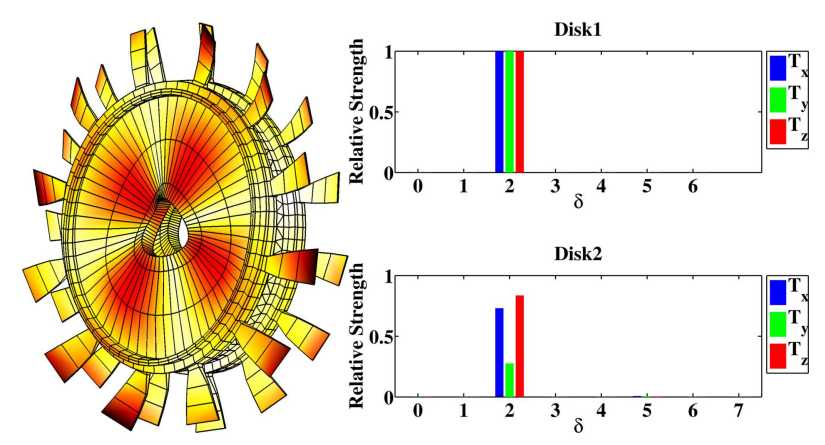

(a)

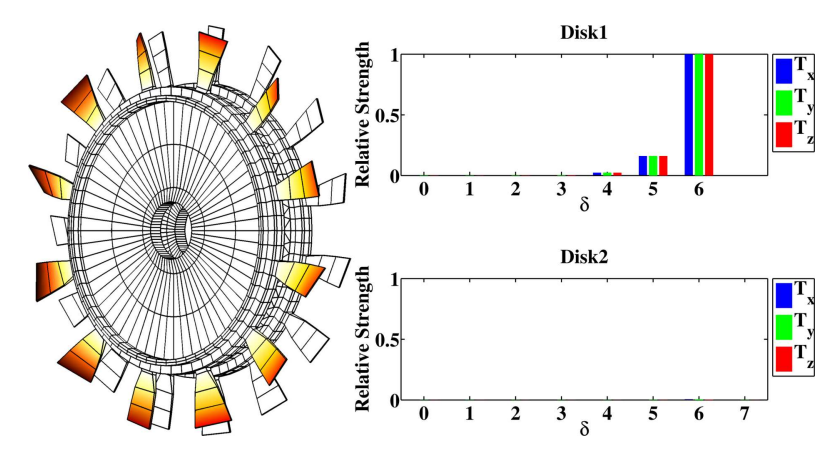

(b)

FIG. 3: (a) Mono-harmonic coupled mode at $f=4.25$ and (b) multi-harmonic localized mode at $f=2.99$ and their harmonic content

as defined by Laxalde et al. ${ }^{8,11}$ are not necessarily modes of the full rotor. In the following section, the theory associated with mono-harmonic solutions is recalled and the error between them and the eigenmodes is qualified.

Let us consider two disks $\mathcal{D}^{1}$ and $\mathcal{D}^{2}$ connected with a volumic interface $\mathcal{R}$ without interior DOF as presented in Fig. 1. The physical displacement is substituted by its Fourier decomposition (Eqn. (8)) in the coupled problem in Eqn. (2). If one considers that any disk $\mathcal{D}^{d}, d \in\{1,2\}$, is tuned, its dynamic stiffness $\left[Z^{d}\right]$ is obtained from that of its reference sector $\left[Z^{d, 0}\right]$ through $\left[Z^{d}\right]=\left[I_{N_{s}^{d}} \otimes Z^{d, 0}\right]$. The projection of the latter matrix onto the subspace 
generated by the Fourier matrix $\left[E^{d^{\top}} \otimes I_{N^{d, 0}}\right]$ is

$$
\begin{aligned}
{\left[\widehat{Z}^{d}\right]=} & {\left[E^{d^{\top}} \otimes I_{N^{d, 0}}\right]\left[I_{N_{s}^{d}} \otimes Z^{d, 0}\right]\left[E^{d} \otimes I_{N^{d, 0}}\right] } \\
= & {\left[E^{d^{\top}} E^{d}\right] \otimes\left[Z^{d, 0}\right] } \\
= & {\left[\begin{array}{ccccc}
N_{s}^{d} Z^{d, 0} & 0 & \cdots & 0 & 0 \\
0 & 2 N_{s}^{d} Z^{d, 0} & \cdots & 0 & 0 \\
\vdots & \vdots & \ddots & \vdots & \vdots \\
0 & 0 & \cdots & 2 N_{s}^{d} Z^{d, 0} & 0 \\
0 & 0 & \cdots & 0 & N_{s}^{d} Z^{d, 0}
\end{array}\right] }
\end{aligned}
$$

Thanks to Eqn (11), the projection of the block-diagonal matrix containing the dynamic stiffnesses of both disks in Eqn. (3) is really straightforward:

$$
\left[\begin{array}{cc}
E^{1} \otimes I_{N^{1,0}} & 0 \\
0 & E^{2} \otimes I_{N^{2,0}}
\end{array}\right]^{\top}\left[\begin{array}{cc}
Z^{1} & 0 \\
0 & Z^{2}
\end{array}\right]\left[\begin{array}{cc}
E^{1} \otimes I_{N^{1,0}} & 0 \\
0 & E^{2} \otimes I_{N^{2,0}}
\end{array}\right]=\left[\begin{array}{cc}
\widehat{Z}^{1} & 0 \\
0 & \widehat{Z}^{2}
\end{array}\right]
$$

$\left[\widehat{Z}^{\mathcal{R}}\right]$ is defined such that

$$
\left[\widehat{Z}^{\mathcal{R}}\right]=\left[\begin{array}{cc}
E^{1} \otimes I_{N^{1,0}} & 0 \\
0 & E^{2} \otimes I_{N^{2,0}}
\end{array}\right]^{\top}\left[Z^{\mathcal{R}}\right]\left[\begin{array}{cc}
E^{1} \otimes I_{N^{1,0}} & 0 \\
0 & E^{2} \otimes I_{N^{2,0}}
\end{array}\right]
$$

If $\left[Z^{\mathcal{R}}\right]$ is decomposed into blocks indexed by $d d^{\prime}$ that couple disks $\mathcal{D}^{d}$ and $\mathcal{D}^{d^{\prime}}$, the blocks indexed by $d d^{\prime}$ in $\left[\widehat{Z}^{\mathcal{R}}\right]$ are then

$$
\left[\widehat{Z}_{d d^{\prime}}^{\mathcal{R}}\right]=\left[E^{d} \otimes I_{N^{d, 0}}\right]^{\top}\left[Z_{d d^{\prime}}^{\mathcal{R}}\right] \quad\left[E^{d^{\prime}} \otimes I_{N^{d^{\prime}, 0}}\right]
$$

The different blocks indexed by $d d^{\prime}$ can themselves be decomposed into blocks indexed by $\delta \delta^{\prime}$ that couple a shape of harmonic $\delta$ on disk $\mathcal{D}^{d}$ to a a shape of harmonic $\delta^{\prime}$ on $\mathcal{D}^{d^{\prime}}$ :

$$
\left[\widehat{Z}_{d d^{\prime}, \delta \delta^{\prime}}^{\mathcal{R}}\right]=\left[E_{\delta}^{d} \otimes I_{N^{d, 0}}\right]^{\top}\left[Z_{d d^{\prime}}^{\mathcal{R}}\right] \quad\left[E_{\delta^{\prime}}^{d^{\prime}} \otimes I_{N^{d^{\prime}, 0}}\right]
$$

This inter-harmonic coupling prevents the mechanical problem from being decomposed into individual subproblems for every harmonic coefficient as it could be done in the mono-disk analysis. This also implies that the free vibration modes of the rotor can only be monoharmonic if the inter-harmonic coupling coefficients in (15) have a negligible influence. In the present formulation, the submatrices indexed by $\delta \delta^{\prime}, \delta \neq \delta^{\prime}$, in the blocks $\left[\widehat{Z}_{d d^{\prime}}^{\mathcal{R}}\right]$ are neglected, so that harmonics are decoupled.

$$
\left.\left[\widehat{Z}_{d d^{\prime}}^{\mathcal{R}}\right]=\left[\widehat{Z}_{d d^{\prime}, \delta \delta}^{\mathcal{R}}\right]+\widehat{Z}_{d d^{\prime}, \delta \neq \delta \mathcal{L}}^{\mathcal{R}}\right]
$$


With this approximation, $\left[\widehat{Z}_{\delta}^{\mathcal{R}}\right]$ is defined retroactively such that

$$
\left[\widehat{Z}_{\delta}^{\mathcal{R}}\right]=\left[\begin{array}{cc}
E_{\delta}^{1} \otimes I_{N^{1,0}} & 0 \\
0 & E_{\delta}^{2} \otimes I_{N^{2,0}}
\end{array}\right]^{\top}\left[Z^{\mathcal{R}}\right]\left[\begin{array}{cc}
E_{\delta}^{1} \otimes I_{N^{1,0}} & 0 \\
0 & E_{\delta}^{2} \otimes I_{N^{2,0}}
\end{array}\right]
$$

The approximation error can be quantified from $\left[\widehat{Z}_{d d^{\prime}, \delta \neq \delta^{\prime}}^{\mathcal{R}}\right]$. In future work, this error estimation will be integrated into a residual iteration technique in order to enrich the subspaces of approximation in the subsequent reduction process.

From the structures of the harmonic matrices in Eqns. (12) and (17), one obtains a class of parametrized problems with respect to $\delta$ :

- if $\delta=0$

$$
\begin{array}{r}
\left(\left[\begin{array}{cc}
N_{s}^{1} Z^{1,0} & 0 \\
0 & N_{s}^{2} Z^{2,0}
\end{array}\right]+\left[\widehat{Z}_{0}^{\mathcal{R}}\right]\right)\left\{\begin{array}{l}
\widehat{q}_{0}^{1} \\
\widehat{q}_{0}^{2}
\end{array}\right\}=\{0\} \\
{\left[\begin{array}{cc}
c_{l}^{1}-c_{r}^{1} & 0 \\
0 & c_{l}^{2}-c_{r}^{2}
\end{array}\right]\left\{\begin{array}{l}
\widehat{q}_{0}^{1} \\
\widehat{q}_{0}^{2}
\end{array}\right\}=\{0\}}
\end{array}
$$

- else

$$
\begin{gathered}
\left.2\left[\begin{array}{cccc}
N_{s}^{1} Z^{1,0} & 0 & 0 & 0 \\
0 & N_{s}^{1} Z^{1,0} & 0 & 0 \\
0 & 0 & N_{s}^{2} Z^{2,0} & 0 \\
0 & 0 & 0 & N_{s}^{2} Z^{2,0}
\end{array}\right]+\left[\widehat{Z}_{\delta}^{\mathcal{R}}\right]\right)\left\{\begin{array}{c}
\Re\left(\widehat{q}_{\delta}^{1}\right) \\
\Im\left(\widehat{q}_{\delta}^{1}\right) \\
\Re\left(\widehat{q}_{\delta}^{2}\right) \\
\Im\left(\widehat{q}_{\delta}^{2}\right)
\end{array}\right\}=\{0\} \\
{\left[\begin{array}{cc}
c_{l}^{1}-c_{r}^{1} e^{2 \mathbf{i} \pi \delta \alpha^{1}} & 0 \\
0 & c_{l}^{2}-c_{r}^{2} e^{2 \mathbf{i} \pi \delta \alpha^{2}}
\end{array}\right]\left\{\begin{array}{c}
\widehat{q}_{\delta}^{1} \\
\widehat{q}_{\delta}^{2}
\end{array}\right\}=\{0\}}
\end{gathered}
$$

The equations that come first in the problems above are not sufficient to define a cyclic modeshape. An observation equation that states how the shapes are dephased between two adjacent sectors is added to each problem. The observation matrices $\left[c_{r}^{d}\right]$ and $\left[c_{l}^{d}\right]$ are used to isolate the right and left interface DOF.

In the second problem, the projection of the dynamic stiffness matrix of any of the disks is equivalent to duplicate this matrix. This is not the case for the ring, which leads to nearpair eigensolutions. For thinner meshes of the ring, the near-pair solutions would converge to exact pairs. Throughout the rest of this paper, only the solutions without aliasing are considered:

$$
\delta \leq \min _{d}\left(\frac{N_{s}^{d}}{2}\right)
$$


This is referred to as "one to one nodal diameters" by Laxalde et al. ${ }^{8,11}$.

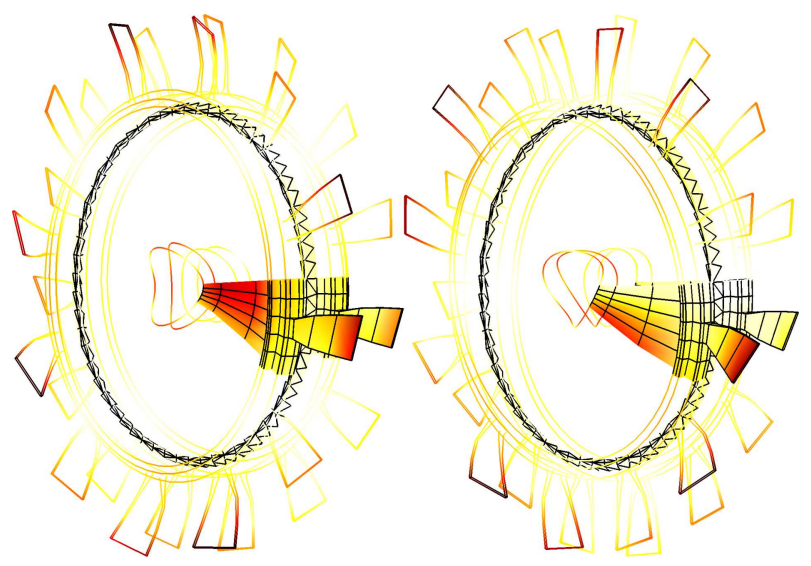

FIG. 4: Near-pair of mono-harmonic solutions with $\delta=2$ at $f=4.25$

As an illustration, the near-pair of eigensolutions at $f=4.25$ with $\delta=2$ is presented in Fig. 4. The chosen representation focuses on the solution that is really computed for a sector of the rotor, then restored to the whole structure.

It seems that they share some common properties as displayed in Figs. 3a and 4. The Modal Assurance Criterion (MAC) is 0.989 for both modes and their frequency relative error is 0.031 and 0.033 respectively. This excellent correlation states that this near-pair of mono-harmonic solutions is locally (in terms of frequency) a near-pair of modes.

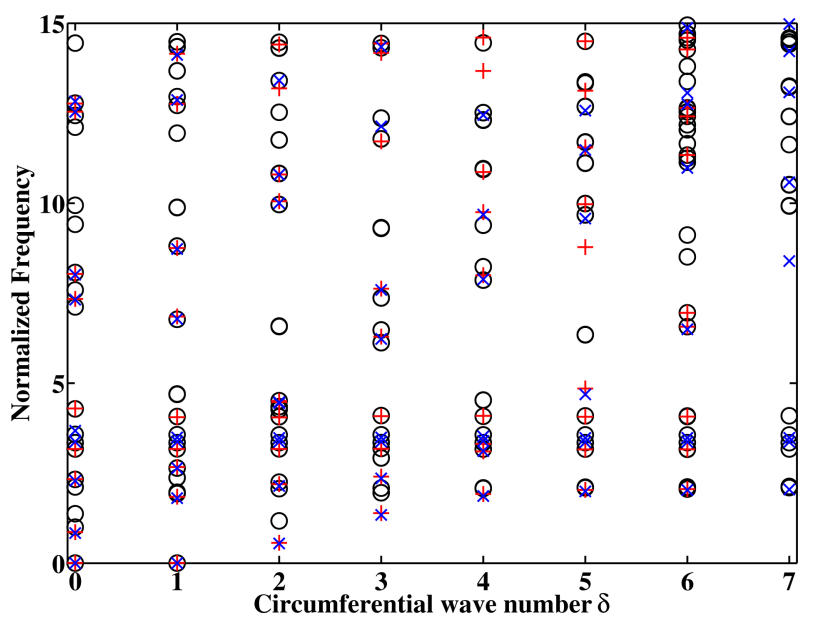

FIG. 5: Normalized frequency vs. nodal diameter diagram for the mono-harmonic eigensolutions of the rotor $(\bigcirc)$ and for the eigenmodes of disks $\mathcal{D}^{1}(+)$ and $\mathcal{D}^{2}(\times)$ in free-free condition

Finally, a classical frequency versus nodal diameter diagram is displayed in Fig. 5 either Paper number GTP-08-1085

A. Sternchüss 
for the eigensolutions of the rotor or the modes of the individual disks when considered free-free. This graph shows significant discrepancies which can be explained by the fact that the inter-stage interfaces are not exactly fixed when the disks are assembled together, but they are not free either. Accurate boundary conditions to apply to individual disks would be somewhere between free and fixed, depending on the modeshape.

\section{Model Reduction}

According to the previous section, mono-harmonic solutions are not modes, but they will be used to approximate the kinematics of the bladed sectors, in order to build a reduced model of the rotor. To do so, each bladed sector is represented as a super-element that allows:

(i) to target a set of eigenmodes from a set of mono-harmonic solutions. Since it is assumed that each eigenmode is the linear combination of mono-harmonic solutions whose frequencies lie in the vicinity of the corresponding natural frequency, the targeting of an eigenmode is in fact the targeting of the narrow frequency range where its harmonic components are found. It is also possible to select a subset of the harmonics a modeshape is made of. In practice, only the mono-harmonic solutions with low $\delta$ are selected, since only these modeshapes account for disk-dominated motion at low frequencies ${ }^{1}$.

(ii) to include blade-dominated motion into the reduced model by adding a set of modes of the individual sectors with their left and right interfaces clamped. Such modeshapes are used since fewer of them are required to account for blade-dominated motion than cyclic modeshapes with high $\delta$ at low frequencies.

\section{Degrees of freedom of the super-elements}

Three types of generalized DOF are assigned to each sector super-element, they are defined in correspondance to a subset of physical DOF:

(i) DOF that belong to the inter-sector elements (green area in Fig. 6),

(ii) DOF that belong to the inter-disk ring (red area in Fig. 6), 
(iii) purely interior DOF (the other DOF).

Generalized DOF sets (i) and (ii) can be joint. In the previous approach ${ }^{9}$, since the gen-

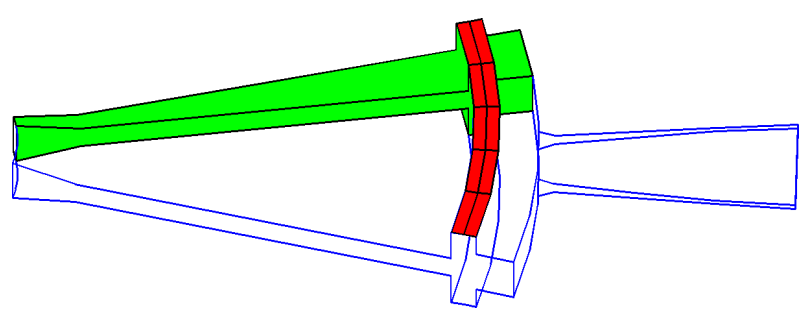

FIG. 6: Sector model: ( $\square$ ) sector super-element, ( $\square$ ) inter-sector super-element and inter-disk ring surface elements

eralized DOF are paired with physical DOF, the compatibility between two clockwisely consecutive sectors was ensured by keeping the physical finite elements with at least one node that belongs to the left interface. In the present updated technique, each slice of intersector elements is also condensed into a super-element whose generalized DOF are those of its two neighbouring sector super-elements, as illustrated in Figs. 6 and 7. The reduced rotor model keeps only the inter-disk ring unchanged.

\section{Subspaces of motion}

Let us consider a sector $\mathcal{S}^{d, s}$ with:

(i) a set of eigenmodes $\left[\Phi_{f i x}^{d, s}\right]$ of this sector with its left and right interfaces both clamped. These modes can differ from a sector to another.

(ii) a set of mono-harmonic solutions $\left[\Re\left(\left[\Phi_{r c y c}^{d, s}\right]\right) \Im\left(\left[\Phi_{r c y c}^{d, s}\right]\right)\right]$ whose harmonics $\delta$ are selected for a particular reason (design, ...). These modeshapes are identical for all the sectors of a given disk.

The initial set of vectors is:

$$
\left[T_{\text {init }}^{d, s}\right]=\left[\left[\Phi_{\text {fix }}^{d, s}\right] \Re\left(\left[\Phi_{\text {rcyc }}^{d, s}\right]\right) \Im\left(\left[\Phi_{\text {rcyc }}^{d, s}\right]\right)\right]
$$

The sorting order of these vectors is critical to ensure a good condition number of the resulting matrices, since iterative procedures of orthonormalization are used to turn the initial set of vectors into the bases of the kinematic subspaces. In the present reduction 


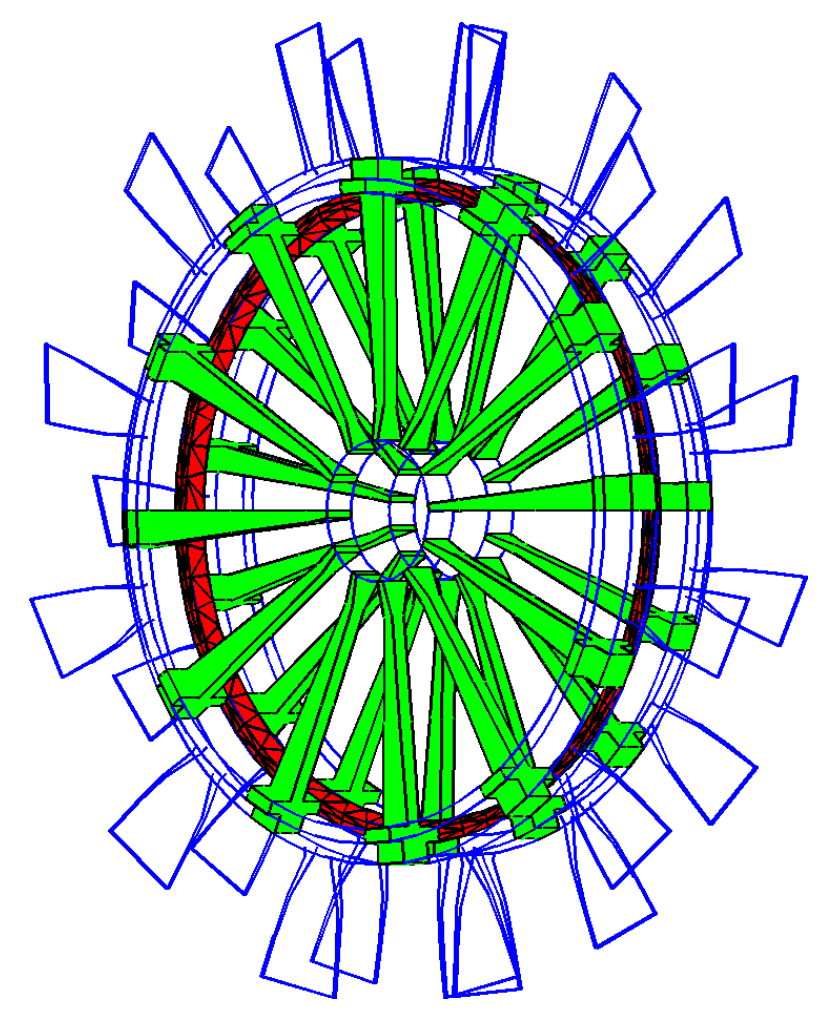

FIG. 7: Reduced rotor model: ( $\square$ ) sector super-elements, ( $\square$ ) inter-sector super-elements and (ם) inter-disk ring elements

process, an "Orthogonal Maximum Sequence" 12 is used to orthogonalize the vectors. The main advantage of the OMS is that it is faster than the standard or modified Gramm-Schmidt procedure. At step $n$ of this algorithm, a sensor is placed at the physical DOF where vector $n$ has its maximum and the latter physical DOF is defined as the generalized DOF associated with vector $n$. The remaining vectors are processed so that their observability is zero with respect to the current sensor. This process is repeated until all sensors are placed. It leads to a basis $\left[T_{M}^{d, s}\right]$ that relates the generalized DOF $\left\{q_{M}^{d, s}\right\}$ to the physical DOF of sector $\mathcal{S}^{d, s}$ thanks to Eqn. (21).

$$
\left\{q^{d, s}\right\}=\left[T_{M}^{d, s}\right]\left\{q_{M}^{d, s}\right\}
$$

Even though it is never explicitely built, the subspace in which the approximate solutions 
are sought is given by Eqn. (22).

$$
\left[T_{M}\right]=\left[\begin{array}{cccccc}
T_{M}^{1,0} & \cdots & 0 & 0 & \cdots & 0 \\
\vdots & \ddots & \vdots & \vdots & & \vdots \\
0 & \cdots & T_{M}^{1, N_{s}^{1}-1} & 0 & \cdots & 0 \\
0 & \cdots & 0 & T_{M}^{2,0} & \cdots & 0 \\
\vdots & & \vdots & \vdots & \ddots & \vdots \\
0 & \cdots & 0 & 0 & \cdots & T_{M}^{2, N_{s}^{1}-1}
\end{array}\right]
$$

and the generalized DOF vector $\left\{q_{M}\right\}$ is related to the physical DOF vector $\{q\}$ thanks to Eqn. (23).

$$
\{q\}=\left[T_{M}\right]\left\{q_{M}\right\}
$$

\section{Assembly of the super-elementary matrices}

Instead of computing the reduced matrices by the direct projection

$$
\left[Z_{M}\right]=\left[T_{M}\right]^{\top}[Z]\left[T_{M}\right]
$$

where $[Z]$ is defined in Eqn. (3), a two-step procedure is followed:

Step 1: the physical finite element matrices of the sectors are projected onto their own kinematic subspace:

$$
\left[Z_{M}^{d, s}\right]=\left[T_{M}^{d, s}\right]^{\top}\left[Z^{d, s}\right]\left[T_{M}^{d, s}\right]
$$

$\left[Z^{d, s}\right]$ is the dynamic stiffness of sector $\mathcal{S}^{d, s}$. These reduced matrices are then assembled with respect to the generalized DOF associated with the super-elements. This operation leads to the reduced matrices of both sector and inter-sector super-elements.

Step 2: the matrices of the ring are projected onto the kinematic subspaces of the neighbouring disks with respect to the inter-disk generalized DOF.

$$
\left[Z_{M}^{\mathcal{R}}\right]=\left[T_{M}\right]^{\top}\left[Z^{\mathcal{R}}\right]\left[T_{M}\right]
$$

In order to avoid using the full matrix of modeshapes $\left[T_{M}\right],\left[Z_{M}^{\mathcal{R}}\right]$ is built iteratively sector by sector. 


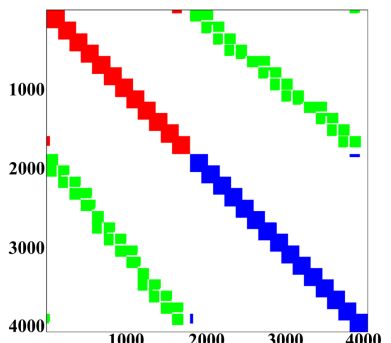

(a)

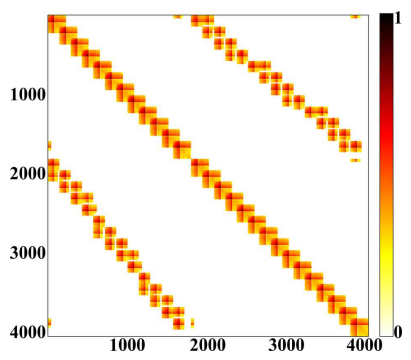

(b)

FIG. 8: Reduced stiffness matrix $\left[K_{M}\right]$ (a) global shape: ( $\square$ ) blocks of disk $\mathcal{D}^{1},(\boldsymbol{\square})$ blocks of disk $\mathcal{D}^{2}$ and $(\square)$ blocks of ring $\mathcal{R}$, and (b) actual matrix

The reduced dynamic stiffness $\left[Z_{M}\right]$ is finally assembled with respect to the full set of generalized DOF.

All the reduced matrices are relatively sparse. Besides, they have the remarkable form displayed in Fig. 8 for the assembly of two tuned disks with 12 and 15 blades. The diagonal blocks and four off-diagonal blocks derive from the projection of the sector matrices. The latter blocks can be divided into two groups, the first with 12 blocks and the second with 15 blocks. The inter-disk coupling is contained both in the diagonal blocks that are derived from the matrices of the inter-sector joints and in the off-diagonal blocks that are derived from the matrices of the ring. Apart from these remarks, the reduced matrices have no other properties which could impact their eigenvalues or eigenvectors.

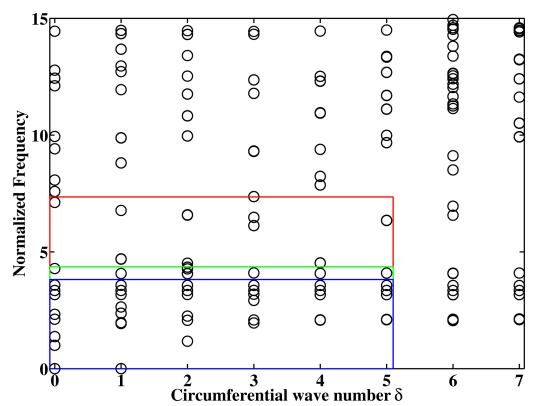

(a)

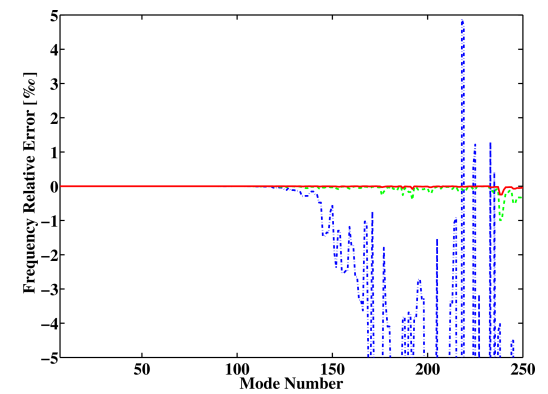

(b)

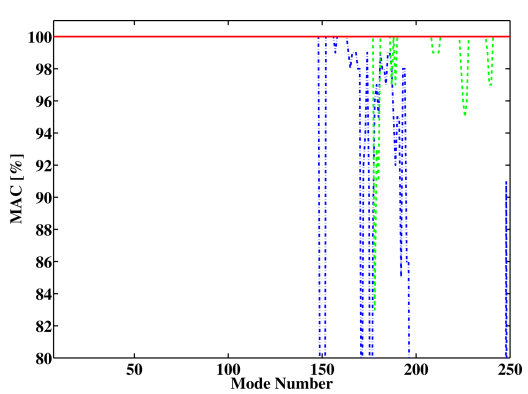

(c)

FIG. 9: (a) Targeted frequency ranges, (b) frequency relative error and (c) modeshape correlation between reference and generalized modes.

Notice that the generalized load can be obtained from the physical load with the same procedures. 


\section{Numerical Results}

Once the reduced matrices and load vectors have been assembled, it is possible to compute the free or forced response of the rotor.

The generalized eigenmodes are solutions of the homogeneous problem:

$$
\left[Z_{M}(\omega)\right]\left\{q_{M}(\omega)\right\}=\{0\}
$$

The physical modeshapes are then restored to the full structure thanks to Eqn. (23). These modeshapes can be compared to those computed with the full model.

In the case of the sample rotor with 12 and 15 blades, different sets of mono-harmonic solutions with $\delta \in\{0,5\}$ were computed for various targeted frequency ranges and were used to reduce the rotor problem, as displayed in Fig. 9a. 250 generalized modes, comprised between $f=0$ and $f=15$, were compared to the assumed corresponding modes of the rotor in the same frequency range, referred to as reference modes. The resulting relative error in frequency is plotted in Fig. 9b. A modeshape correlation index, based on a Modal Assurance Criterion (MAC) is reported in Fig. 9c. As can been seen in these figures, the maximum of the relative error in frequency is below $0.1 \%$ and the modeshape correlation is excellent as soon as subspace $\left[T_{M}\right]$ includes enough vectors. A future development of this work is to define a priori error estimators, probably based on the identified source of error underlined in the mono-harmonic computation section.

\section{APPLICATION TO AN INDUSTRIAL MODEL}

The industrial model to which the methodology described in the previous section is applied is a part of the numerical model of a compressor in development, it is depicted in Fig. 10. It is composed of three integrally bladed disks (blisks), whose characteristics are reported in Tab. I. The full finite element model of the assembly is made of 11, 289, 230 tetrahedrons connecting 2,631,094 nodes, which leads to 7,893, 282 DOF. The material of the stages are special alloys of titanium. Since the finite element model is quite huge as is, only linear elements were considered at the time of the present study, but computations with quadratic elements are planned.

As described in the beginning of this paper, one uses inter-disk rings built from a Delaunay tessellation between the nodes of the regarding rims of two adjacent disks. Since the nominal 


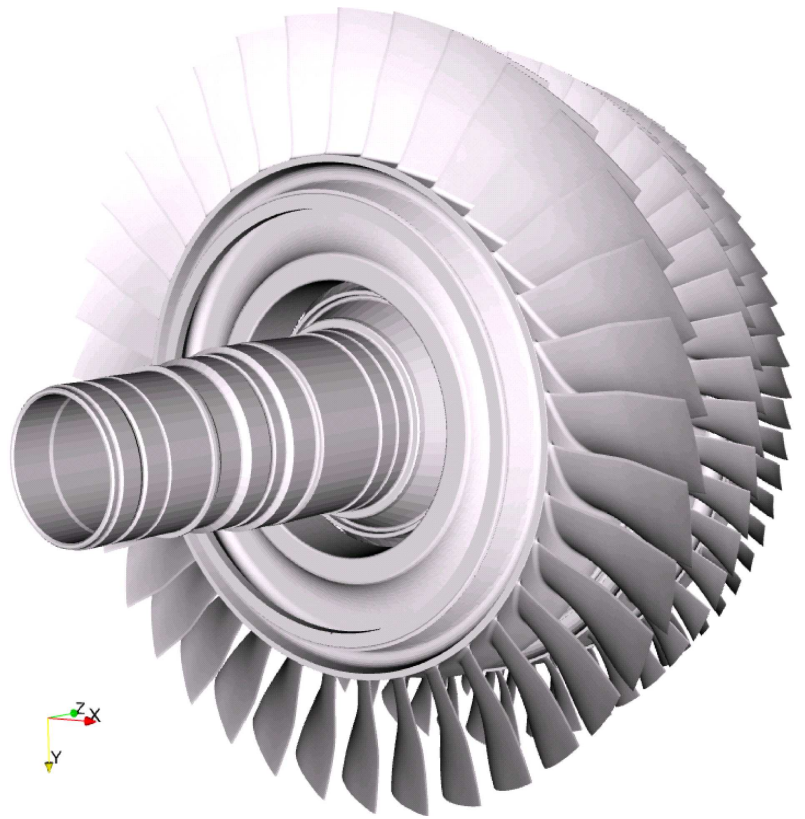

(a)

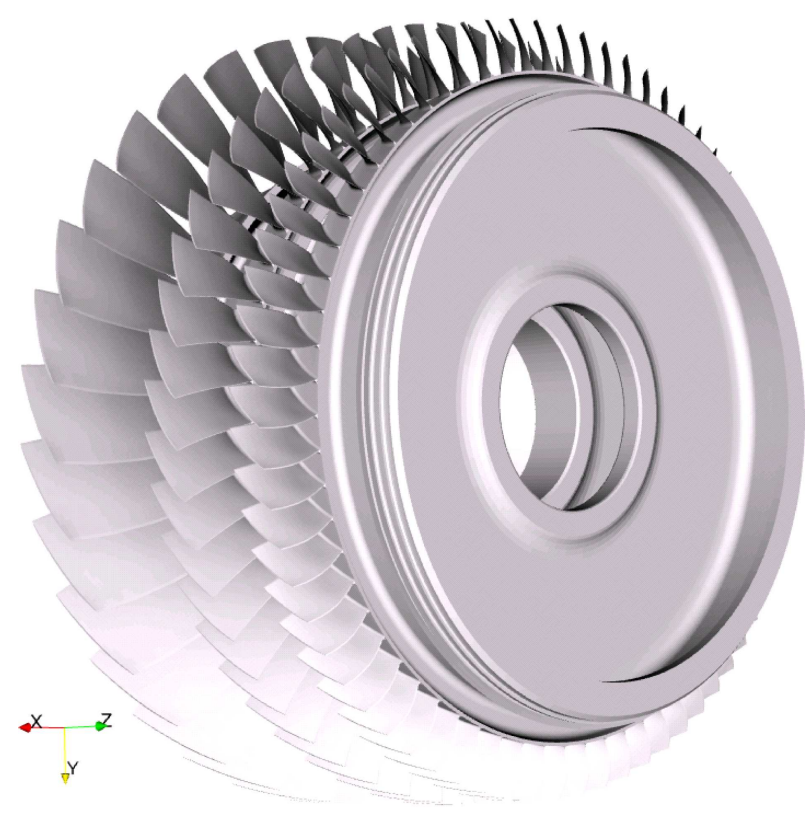

(b)

FIG. 10: Industrial 3 stage HP compressor: (a) front and (b) rear.

TABLE I: Description of the finite element model

\begin{tabular}{|c|c|c|c|c|c|}
\hline Stage & $\begin{array}{c}\text { Nb. of } \\
\text { sectors }\end{array}$ & $\begin{array}{c}\text { Nb. of } \\
\text { Nodes/sec. }\end{array}$ & $\begin{array}{c}\text { Nb. of } \\
\text { Elements/sec. }\end{array}$ & $\begin{array}{c}\text { Nb. of } \\
\text { DOF/sec. }\end{array}$ & $\begin{array}{c}\text { Type of } \\
\text { Elements }\end{array}$ \\
\hline 1 & 36 & 16,434 & 65,170 & 49,302 & Tetra4 \\
\hline 2 & 56 & 9,566 & 36,815 & 28,698 & Tetra4 \\
\hline 3 & 70 & 23,104 & 97,860 & 69,312 & Tetra4 \\
\hline
\end{tabular}

geometries of the sectors of two connected stages are such that they are bonded together, a pre-processing of the geometries is required to slightly translate the nodes of the flanges of disks $\mathcal{D}^{1}$ and $\mathcal{D}^{3}$ so that the inter-disk rings can fill the empty spaces created between the flange of disk $\mathcal{D}^{1}$ (resp. disk $\mathcal{D}^{3}$ ) and the rim of disk $\mathcal{D}^{2}$, such as depicted in Fig. 11. Two sets of nodes have to be moved for each flange:

- the first set is translated longitudinally,

- the second set is translated radially.

This operation does not affect the integrity of the finite element mesh of such parts. Notice 
that the bolting system is not included in the model. The minimal set of elements to

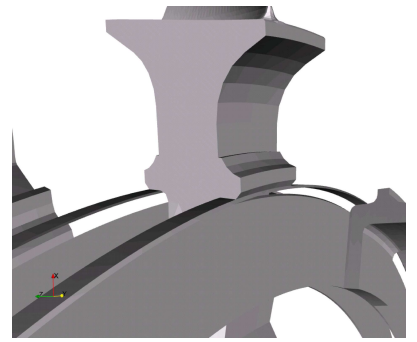

(a)

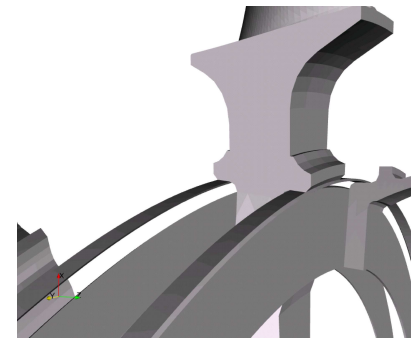

(b)

FIG. 11: Details of the rings (a) between $\mathcal{D}^{1}$ and $\mathcal{D}^{2}$ and (b) between $\mathcal{D}^{2}$ and $\mathcal{D}^{3}$

describe the geometry of the full assembly is presented in Fig. 12, since the bladed disks can be generated by circular repetitions of their initial sector. This allows huge memory savings because only this minimal geometry is stored.

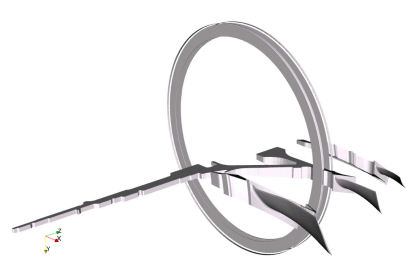

(a)

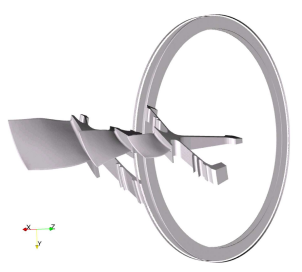

(b)

FIG. 12: Sector models and rings used in the computation of cyclic modeshapes (a) front and (b) rear

\section{Mono-harmonic shapes}

The latter minimal geometry is perfectly suited for the computation of the cyclic modeshapes. A classical graph of normalized frequencies versus nodal diameter is plotted in Fig. 13. It compares the cyclic solutions of the assembly to the modes of the individual disks in free-fix $\left(\mathcal{D}^{1}\right)$, fix-fix $\left(\mathcal{D}^{2}\right)$ and fix-free $\left(\mathcal{D}^{3}\right)$ conditions. Frequencies are normalized with respect to the frequency of the first flexible 0 diameter solution of the rotor.

From Fig. 13, it is noticed that coupled solutions are found inside a triangle that has $\delta=0, f=0$ as a summit, and $\delta=0$ and $f=\delta$ as edges (when $f$ is normalized, as stated before). In this region of high to moderate disk coupling as $\delta$ increases, there are 
noticeable differences between the frequencies of the rotor solutions and the disk natural frequencies. This comes from the fact that a fix condition at inter-stage interfaces is too stiff and disk modes that are similar to solutions of the rotor are higher in frequency. On the contrary, modes of the disks with free rims would be below rotor solutions since a free condition at the inter-stage interfaces is far too soft. Ideal boundary conditions to apply to a mono-disk analysis so that multi-stage effects are properly described would be between free and fix condition, and they would vary according to the considered diameter solution.

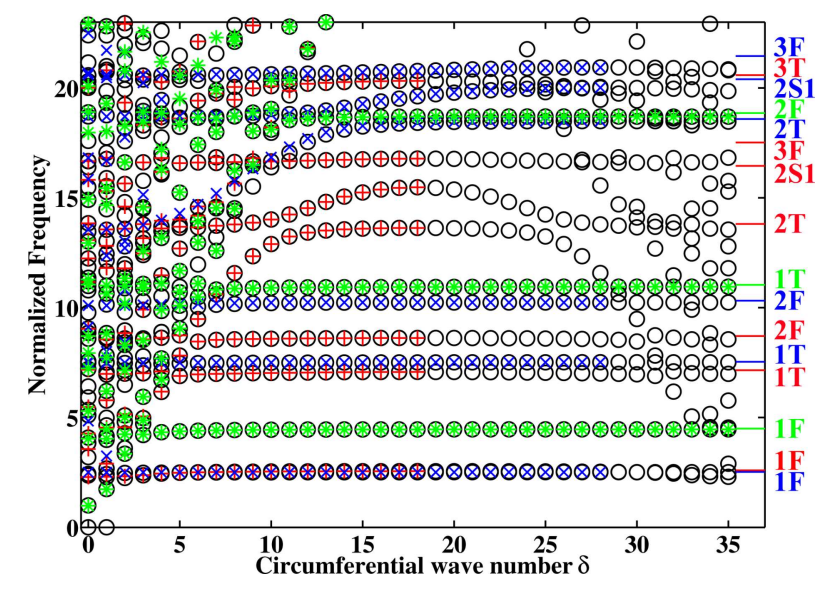

FIG. 13: Normalized frequencies of the cyclic solutions for the rotor $(\bigcirc)$ and the modes of disk $\mathcal{D}^{1}(+)$, disk $\mathcal{D}^{2}(\times)$ and disk $\mathcal{D}^{3}(*)$ in free-fix, fix-fix and fix-free condition. (-), $(-)$ and $(-)$ are frequencies of single sectors with left and right interfaces fixed

Everywhere else, it can be seen that many mono-harmonic solutions are close to modes of the individual disks, merely due to:

(i) a small disk-blade coupling, which tends to confine the strain energy on the blades ${ }^{1}$. In this case, the global motion is very similar to that of the individual disks whatever their boundary conditions are, since the latter do not affect the blade-dominated motion due to the low coupling ratio between the disk and the blades.

(ii) a small disk-disk coupling, which tends to confine the strain energy on a single disk. Besides, a phenomenon of aliasing occurs for mono-harmonic solutions that have a strong participation of disk $\mathcal{D}^{1}$ (resp. $\mathcal{D}^{2}$ ). Typical modeshapes are plotted in Figs. 14 to 17. Notice that the computation of the strain energy in Fig. 14b provides useful information since the maximum of strain energy is located at the rim between disks $\mathcal{D}^{1}$ and $\mathcal{D}^{2}$ and not at the tip of the blades as expected because of the 2S1 shape. 


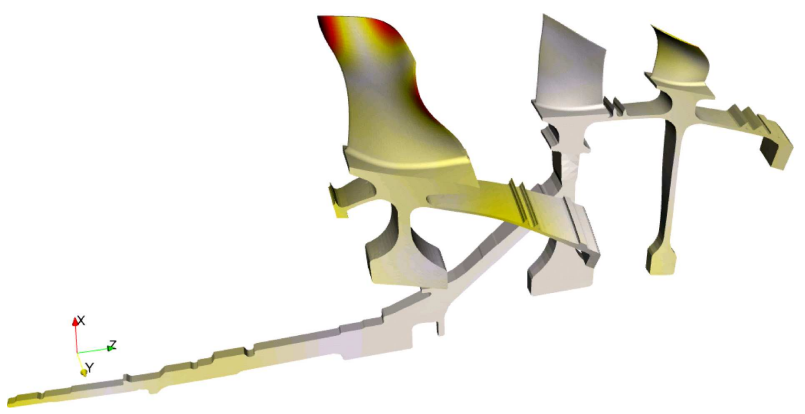

(a)

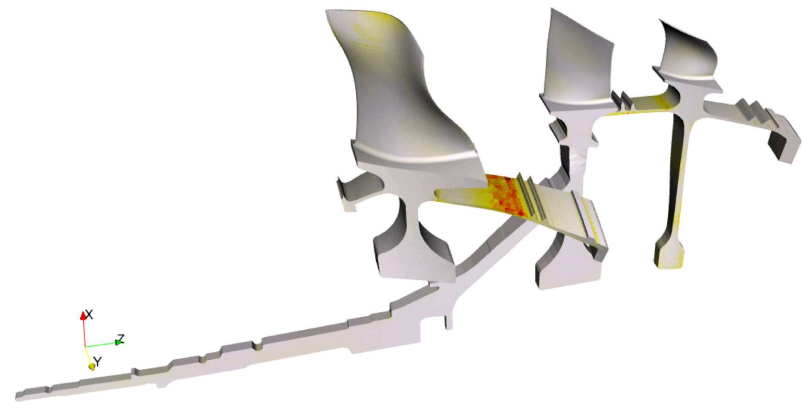

(b)

FIG. 14: Coupled cyclic solution with $\delta=1$ at $f=15.53$ : (a) modeshape and (b) density of strain energy. Blade shapes are 2S1, $2 \mathrm{~F}$ and $1 \mathrm{~T}$.

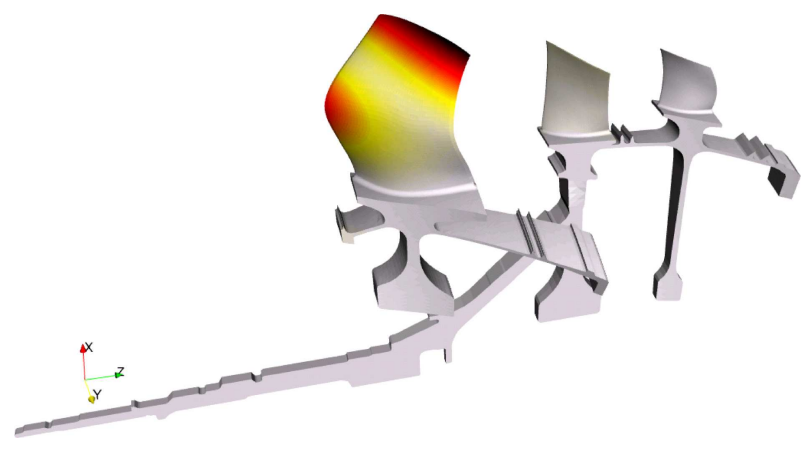

FIG. 15: Cyclic solution localized to disk $\mathcal{D}^{1}$ with $\delta=4$ at $f=8.63$. Blade shape is 2 F.

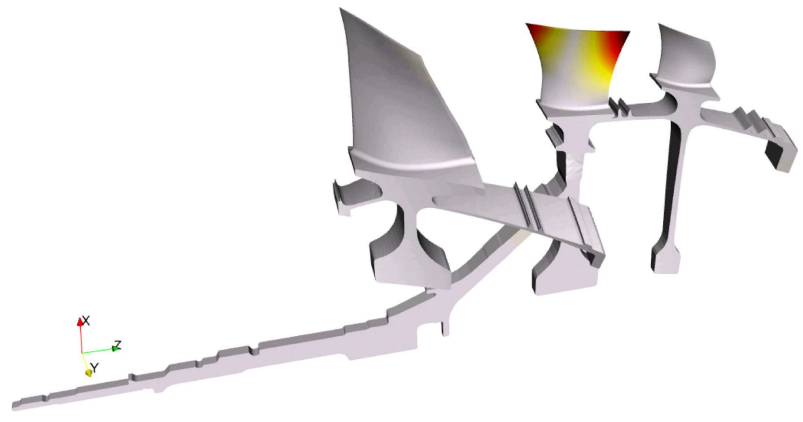

FIG. 16: Cyclic solution localized to disk $\mathcal{D}^{2}$ with $\delta=2$ at $f=7.47$. Blade shape is 1T.

\section{Model Reduction and Results}

A reduced model of this assembly was built from a set of cyclic solutions with $\delta \in\{0,2\}$ and $f \in[0,16.13]$. This reduced model has 44,342 generalized DOF. To capture all the modes in the normalized frequency range [0,19.5], 900 modes were computed. Before restor- 


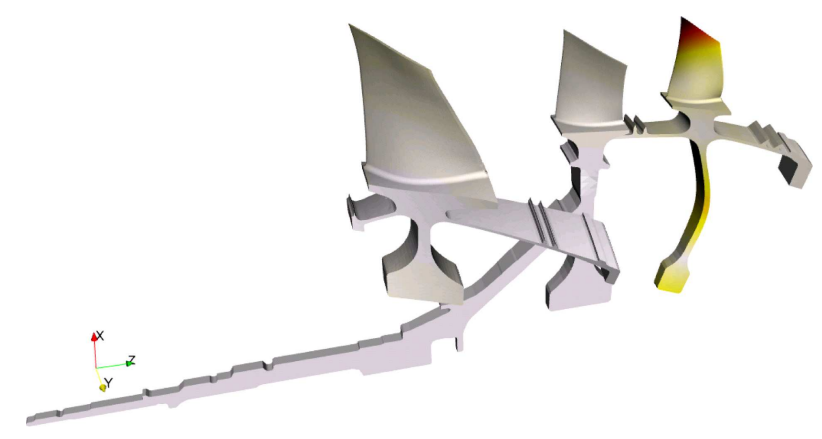

FIG. 17: Cyclic solution localized to disk $\mathcal{D}^{3}$ with $\delta=0$ at $f=5.35$. Blade shape is $1 \mathrm{~F}$.

ing the modeshapes to the whole structure or a subset of physical DOF, a convenient way to describe the generalized modes is to perform a Fourier spatial analysis of the reduced modeshapes. Working with the reduced modeshapes instead of the full modeshapes is possible because without mistuning all the super-elements of a given disk share the same kinematic subspace. Figures 18, 19 and 20 in which the color varies from white (harmonic relative amplitude equals 0) to black (harmonic relative amplitude equals 1) then show that, as expected, modes come first by near-pairs, and second by families.

The participation factor on each disk, in the sense of Eqn. (27), is plotted in parallel to these spectra.

$$
\forall d \in\{1,2,3\}, R^{d}=\frac{\left\{q_{M}^{d}\right\}^{\top}\left[K_{M}^{d}\right]\left\{q_{M}^{d}\right\}}{\left\{q_{M}\right\}^{\top}\left[K_{M}\right]\left\{q_{M}\right\}}
$$

It is mandatory to examine both graphs together to avoid hasty conclusions in terms of multi-harmonicity of modes. Indeed, a mode that looks multi-harmonic on a given disk can be so if its participation relative to that disk is very small: the analysis of an almost zero signal leads to a spectrum that shows multiple harmonics, but it does not indicate that the generalized displacement is almost zero on this disk! Special attention must be paid to the fact that a physical indicator, such as the kinetic energy, should be used to avoid normalization problems between the kinematic subspaces defined on each disk. For instance, modes $118-180$ are clearly localized to disk $\mathcal{D}^{3}$ according to Fig. 18 but seem to be multi-harmonic on disks $\mathcal{D}^{1}$ and $\mathcal{D}^{2}$. The only multi-harmonic modes of the reduced model are observed on disk $\mathcal{D}^{3}$ for $\delta>22$ (if $\delta$ is considered as the dominating harmonic in these particular cases), as described in Fig. 21 where the shape of mode number 428 at $f=10.23$ is restored to the whole assembly, and its weighted spectrum in the sense of Eqn. (10) is plotted. From the latter figures, it can be concluded that almost all modes are 
mono-harmonic, like that of Fig. 22.

\section{Recovery of Modeshapes and Post-processing}

Post-processing the restored modeshapes on the full rotor would be a very expensive numerical effort, since storing the 900 generalized modes represents about $305 \mathrm{Mb}$, whereas storing the 900 modes once restored represents $53 \mathrm{~Gb}$. A procedure of partial recovery of the modeshapes has been developed. Given a selection of elements where the quantities of interest (energies, stresses) are assumed to be located, the generalized response is restored to the physical DOFs of these elements only. These locations can be given:

- either by a spatial Fourier analysis of the generalized displacement or the strain energy associated with each super-element, which leads to recoveries to full sector models,

- or if the generalized displacement appears to be mono-harmonic, a procedure of pairing between mono-harmonic solutions and generalized modes (not described here) returns useful information where to look for stresses or energies.

As an illustration, the generalized mode at $f=15.89$ can be manually paired with the mono-harmonic solution with $\delta=1$ at $f=15.53$ depicted in Fig. 14. Figure 23 displays the strain energy and the Von Mises stress distributions on a subset of physical finite elements that belong to disk $\mathcal{D}^{1}$. This figure shows that, for this generalized mode and this disk, the Von Mises stress is maximum on the disk and, as expected, has a high value in the middle of the blade tip due to the 2S1 shape. Nevertheless, the mono-harmonic solution has a strong participation of disk $\mathcal{D}^{2}$ and a recovery extended to that disk should be computed. 


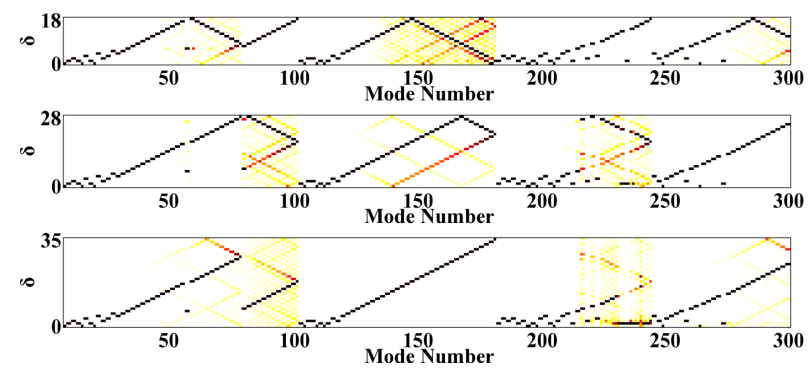

(a)

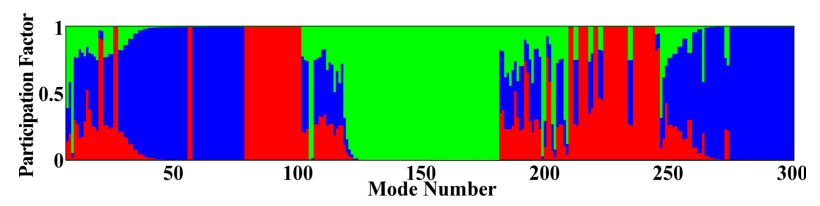

(b)

FIG. 18: Generalized modes 7 to $300(f \in[1,7.52])$ : (a) Spatial harmonic content (b) Participation factor in strain energy for disks $(\square) \mathcal{D}^{1},(\boldsymbol{\square}) \mathcal{D}^{2}$ and $(\square) \mathcal{D}^{3}$

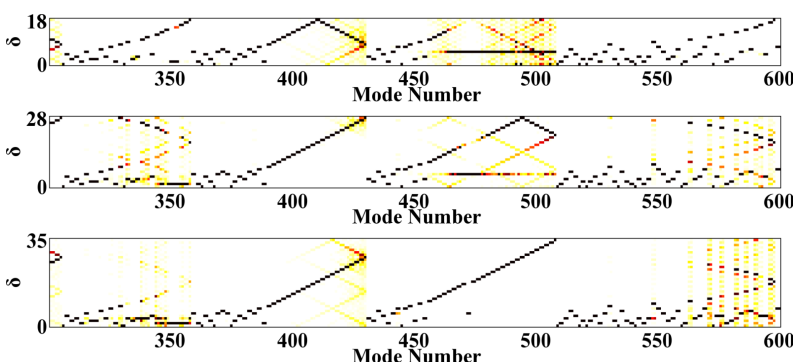

(a)

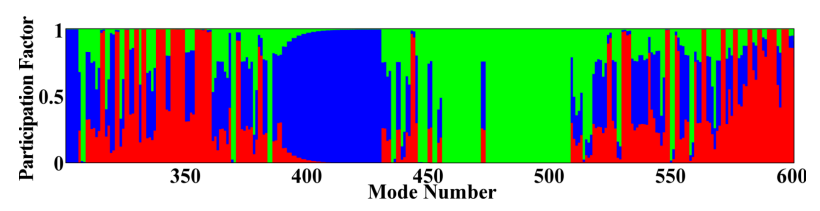

(b)

FIG. 19: Generalized modes 301 to $600(f \in[7.52,13.74])$ : (a) Spatial harmonic content (b) Participation factor in strain energy for disks $(\square) \mathcal{D}^{1},(\mathbf{\square}) \mathcal{D}^{2}$ and $(\square) \mathcal{D}^{3}$ 


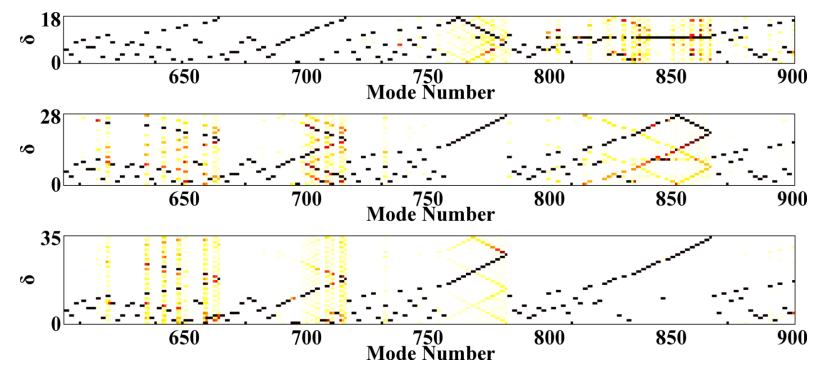

(a)

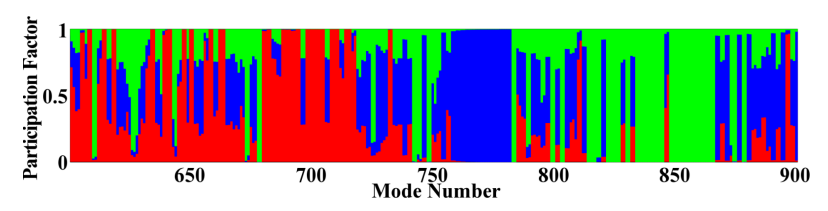

(b)

FIG. 20: Generalized modes 601 to $900(f \in[13.76,19.57])$ : (a) Spatial harmonic content (b) Participation factor in strain energy for disks $(\square) \mathcal{D}^{1},(\mathbf{\square}) \mathcal{D}^{2}$ and $(\square) \mathcal{D}^{3}$ 


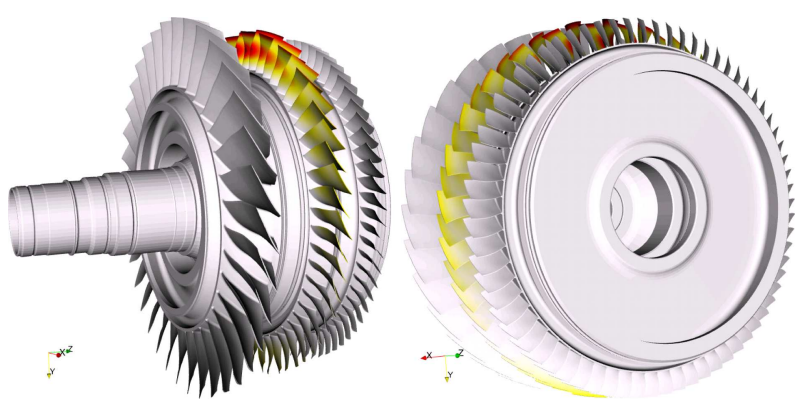

(a)
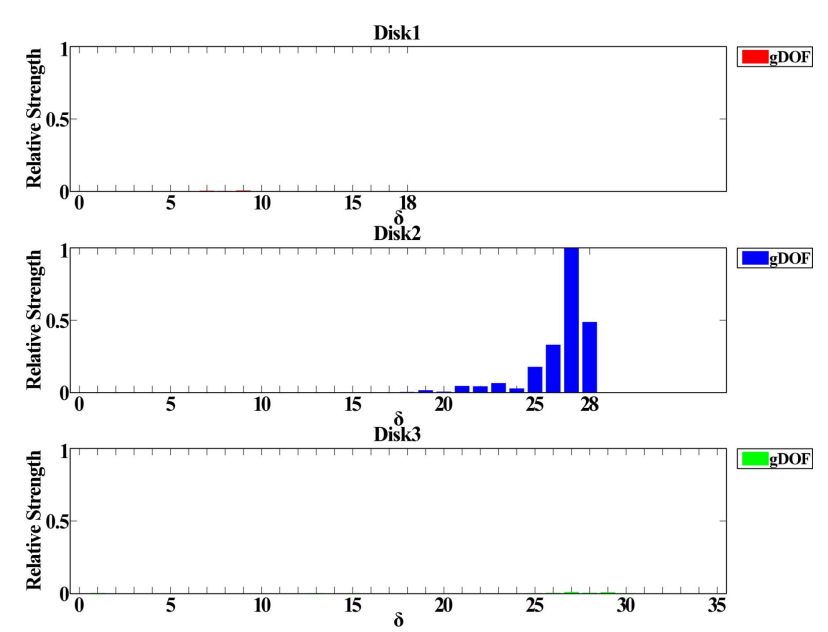

(b)

FIG. 21: Restored mode at $f=10.23$ localized to disk $\mathcal{D}^{2}$ : (a) modeshape and (b) spatial harmonic content for disks $(\square) \mathcal{D}^{1},(\boldsymbol{\square}) \mathcal{D}^{2}$ and $(\square) \mathcal{D}^{3}$ 


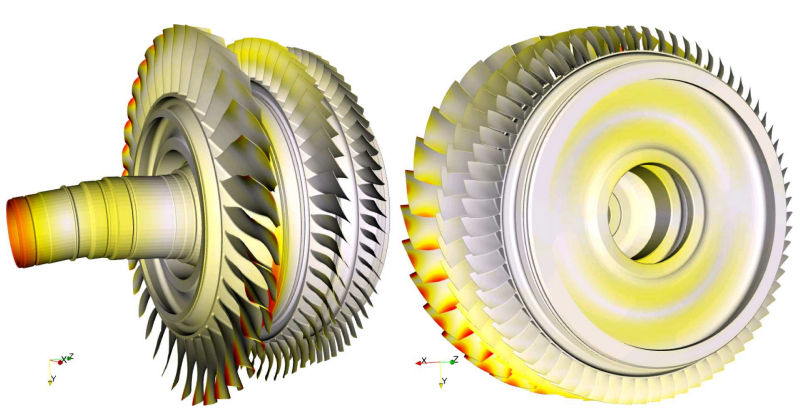

(a)
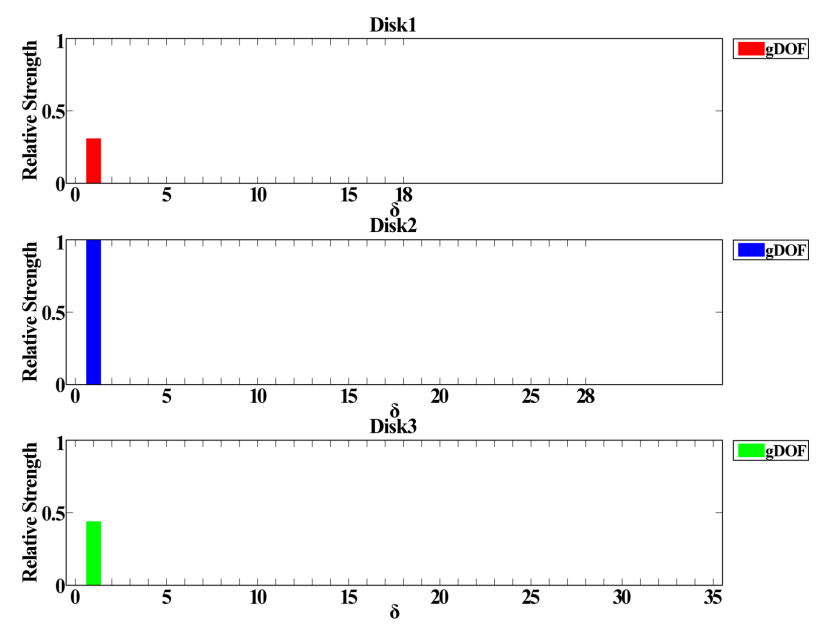

(b)

FIG. 22: Restored mode at $f=15.89$ with strong coupling: (a) modeshape and (b) spatial harmonic content for disks $(\square) \mathcal{D}^{1},(\boldsymbol{\square}) \mathcal{D}^{2}$ and $(\square) \mathcal{D}^{3}$ 

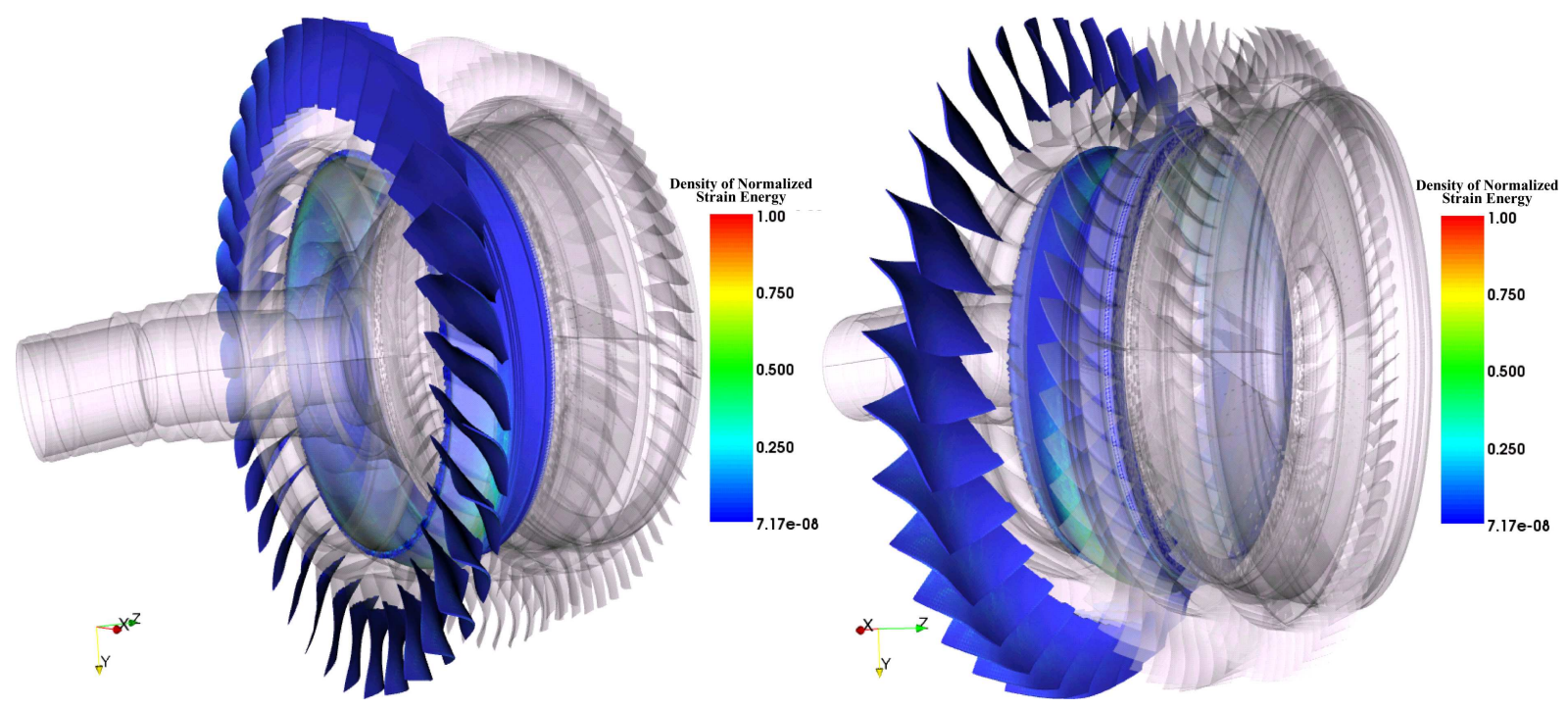

(a)
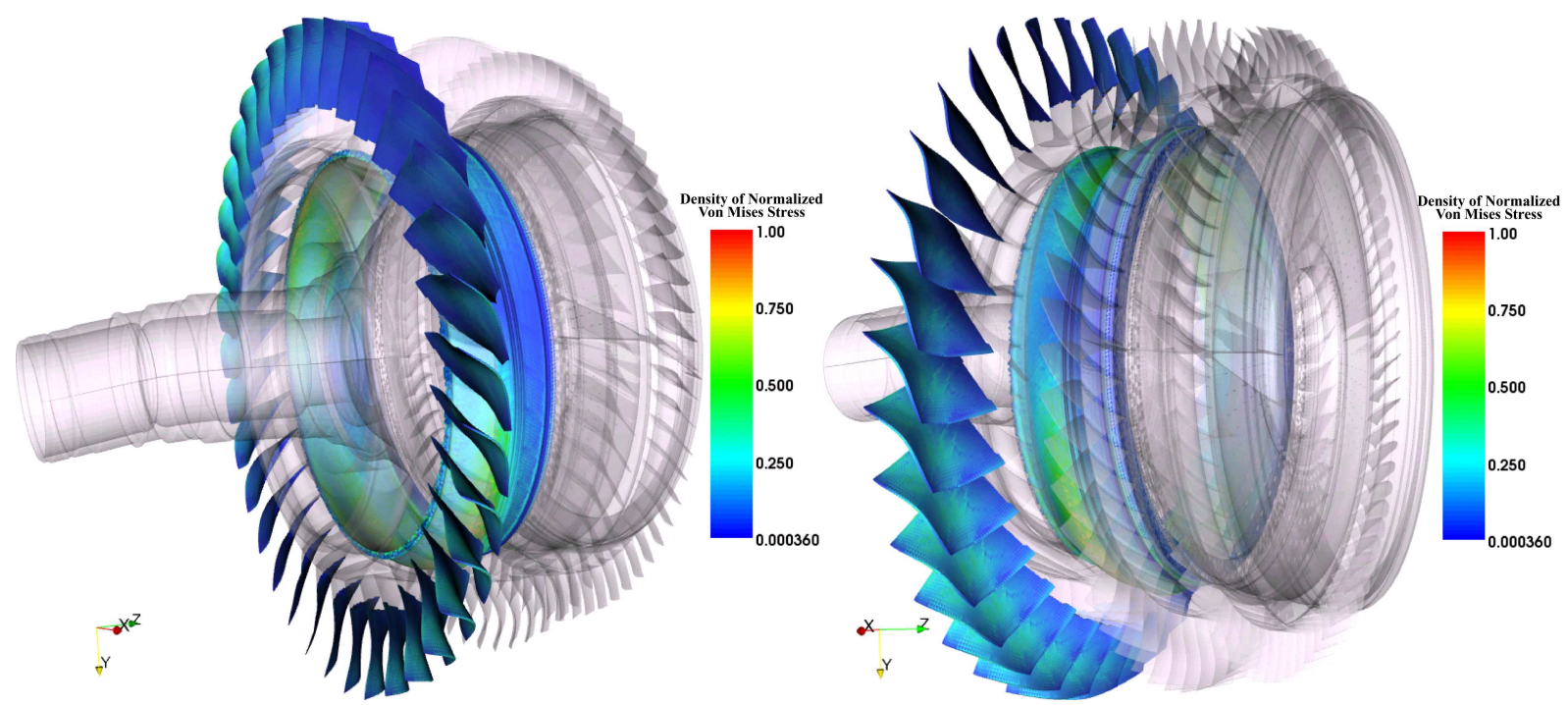

(b)

FIG. 23: Partial post-processing of the generalized mode at $f=15.89$ on disk $\mathcal{D}^{1}$ : (a) normalized density of strain energy and (b) normalized distribution of Von Mises stress 


\section{CONCLUSION}

In the present paper, the authors updated then applied the reduction technique they developed to deal with multi-stage assemblies of bladed disks ${ }^{9}$ to a realistic rotor model. The results obtained for this industrial rotor allow to give some guidelines to use efficiently the previous method in a real industrial environment:

(i) In the proposed methodology, a set of mono-harmonic solutions are computed. Such solutions already contain much valuable information in terms of coupling or regions of high density of strain energy or high stress levels.

(ii) These solutions are used to build a compact reduced model that represents the multistage model with a controlled accuracy. In future work, the error estimator derived from the mono-harmonic computations will be integrated into an iterative procedure of enrichment of the subspaces built to approximate the kinematics of the sectors.

(iii) On the one hand, if a spectral analysis shows that the generalized modes of interest are spatially mono-harmonic, it means that they potentially correspond to monoharmonic solutions, given by a manual or automatic pairing process. This property will be exploited in future work, especially in forced response computations.

(iv) On the other hand, if some of the modes of interest are multi-harmonic, the response is computed with the reduced model.

(v) In every case, the response can be restored to a subset of elements chosen either from an a priori analysis of the mono-harmonic solutions or from an a posteriori analysis of the spatial spectrum of the generalized modes.

The proposed methodology is already implemented within the Structural Dynamics Toolbox $^{13}$ with critical use of the super-element capabilities of this library. Internal element formulations are used although imports from SAMCEF and NASTRAN have also been done for verification. The SDT switch to use NASTRAN as the eigenvalue solver for SDT based models was sometimes used for very large models. The SDT/OpenFEM export to VTK format was used to generate some of the figures included in this paper with ParaView. Developments specific to this study, grouped as the embryo of an SDT/Rotor package, are 
(i) pre-processing tools to assemble sector models and mesh rings using an automated volume mesher,

(ii) mono-harmonic rotor computations,

(iii) sector super-element building from a set of mono-harmonic multi-stage solutions and fixed sector modes,

(iv) post-processing tools for harmonic and generalized coordinate viewing.

\section{Acknowledgments}

Thanks go to Snecma for its technical and financial support. This work takes place in the framework of the MAIA mechanical research and technology program sponsored by CNRS, ONERA and SAFRAN Group.

1 Castanier, M. P., and Pierre, C., 2006. "Modeling and Analysis of Mistuned Bladed Disk Vibration: Status and Emerging Directions". Journal of Propulsion and Power, 22(2), pp. 384396.

2 Lim, S. H., Castanier, M. P., and Pierre, C., 2004. "Vibration Modeling of Bladed Disks Subject to Geometric Mistuning and Design Changes". 45th AIAA/ASME/ASCE/AHS/ASC Structures, Structural Dynamics and Materials Conference.

3 Feiner, D. M., and Griffin, J. H., 2004. "Mistuning identification of bladed disks using a fundamental mistuning model - Part I: Theory". ASME Journal of Turbomachinery, 126, pp. $150-158$.

4 Feiner, D. M., and Griffin, J. H., 2004. "Mistuning identification of bladed disks using a fundamental mistuning model - Part II: Application". ASME Journal of Turbomachinery, 126, pp. 159-165.

5 Sternchüss, A., and Balmès, E., 2006. "On the reduction of quasi-cyclic disks with variable rotation speeds". In Proceedings of the International Conference on Advanced Acoustics and Vibration Engineering (ISMA), pp. 3925-3939. 
6 Bladh, R., Castanier, M. P., and Pierre, C., 2003. "Effects of Multistage Coupling and Disk Flexibility on Mistuned Bladed Disk Dynamics". ASME Journal of Engineering for Gas Turbines and Power, 125, pp. 121-130.

7 Song, S. H., Castanier, M. P., and Pierre, C., 2007. "System Identification of Multistage Turbine Engine Rotors". In Proceedings of ASME Turbo Expo, Montréal, Canada. Paper Number GT2007-28307.

8 Laxalde, D., Lombard, J.-P., and Thouverez, F., 2007. "Dynamics of Multi-Stage Bladed Disk Systems". In Proceedings of ASME Turbo Expo, Montréal, Canada. Paper Number GT200727083.

9 Sternchüss, A., and Balmès, E., 2007. "Reduction of Multistage Rotor Models Through Cyclic Modeshapes". In Proceedings of ASME Turbo Expo, Montréal, Canada. Paper Number GT2007-27974.

10 Ewins, D. J., 1973. "Vibration characteristics of bladed disk assemblies". Journal of Mechanical Engineering Science, 15(3), pp. 165-186.

11 Laxalde, D., Dupeux, J., and Lombard, J.-P., 2006. "Analyse Dynamique des Structures Cycliques Multi-Étages". In Actes du XVè Colloque Vibrations, Chocs et Bruit.

12 Balmès, E., 2005. "Orthogonal Maximum Sequence Sensor Placements Algorithms for modal tests, expansion and visibility". International Modal Analysis Conference.

13 Balmès, E., Bianchi, J.-P., and Leclère, J.-M., 2007. Structural Dynamics Toolbox 6.0 (for use with MATLAB). SDTools, Paris, France. 


\section{List of Figures}

1 Sample assembly . . . . . . . . . . . . . . . . . . 5 5

2 Spatial spectrum of the sample rotor . . . . . . . . . . . . 7

3 Mono- and multi-harmonic modes . . . . . . . . . . . . . . . . 8

4 Near-pair of mono-harmonic solutions . . . . . . . . . . . . . . . . . . . 11

$5 \quad$ Normalized frequency vs. nodal diameter diagram for the mono-harmonic eigensolutions of the rotor and for the eigenmodes of the disks . . . . . . . 11

6 Sector super-elements . . . . . . . . . . . . . . . . . 13

$7 \quad$ Reduced rotor model . . . . . . . . . . . . . . . . . . . . . 14

8 Reduced stiffness matrix . . . . . . . . . . . . . . . . . . 16

9 Frequency and Modeshape Correlation . . . . . . . . . . . . . . . 16

10 Industrial HP compressor . . . . . . . . . . . . . . . . . . . 18

11 Details of the rings . . . . . . . . . . . . . . . . . . . . 19

12 Sector models and rings used in the computation of cyclic modeshapes . . . 19

13 Normalized frequencies of the cyclic solutions for the rotor and of the modes of the disks . . . . . . . . . . . . . . . . . . . . 20

14 Coupled cyclic solution with $\delta=1$ at $f=15.53 \ldots \ldots$. . . . . . . 21

15 Cyclic solution localized to disk $\mathcal{D}^{1}$ with $\delta=4$ at $f=8.63 \ldots \ldots$. . . . 21

16 Cyclic solution localized to disk $\mathcal{D}^{2}$ with $\delta=2$ at $f=7.47 \ldots \ldots$. . . . 21

17 Cyclic solution localized to disk $\mathcal{D}^{3}$ with $\delta=0$ at $f=5.35 \ldots \ldots$. . . . 22

18 Generalized modes 7 to 300 . . . . . . . . . . . . . . . . . 24

19 Generalized modes 301 to 600 . . . . . . . . . . . . . . . . . . . . 24

20 Generalized modes 601 to 900 . . . . . . . . . . . . . . 25

21 Restored mode at $f=10.23$ localized to disk $\mathcal{D}^{2} \ldots \ldots \ldots$. . . . . . . 26

22 Restored mode at $f=15.89$ with strong coupling . . . . . . . . . . . 27

23 Partial post-processing of the generalized mode at $f=15.89$ on disk $\mathcal{D}^{1}$. . 28

\section{List of Tables}

I Description of the finite element model . . . . . . . . . . . . . . . . 18 